\title{
Extreme compression behaviour of higher derivative properties of solids based on the generalized Rydberg equation of state
}

\author{
J.Shanker, B.P.Singh, K.Jitendra \\ Department of Physics. Institute of Basic Sciences, Khandari, Agra, 282002, India \\ Received September 24, 2008, in final form April 2, 2009 \\ We have derived formulations for the pressure derivatives of bulk modulus up to the third order and for higher \\ order Grüneisen parameters using the generalized free volume theory, and the generalized Rydberg equation \\ of state. The properties derived in the present study are directly related to the understanding of thermoelastic \\ properties of solids. The third order Grüneisen parameter (lambda $\lambda$ ) in the limit of infinite pressure has \\ been found to approach a positive finite value for lambda infinity $\left(\lambda_{\infty}\right)$ equal to $1 / 3$. This is a result shown \\ to be independent of the value of K-prime infinity, i. e., the pressure derivative of the bulk modulus at infinite \\ pressure. The results based on other equations of state have also been reported and discussed. We find a \\ relationship between $\lambda_{\infty}$ and pressure derivatives of bulk modulus at infinite pressure which is satisfied by \\ different types of equations of state.
}

Key words: pressure derivatives of bulk modulus, Grüneisen parameters, infinite pressure behaviour, thermoelastic properties

PACS: $65,64.10 .+h, 91.60 . F e, 46.25 .4 f, 62.20 . D, 81.40 . J j, 62.50 .-p$

\section{Introduction}

The Vinet equation of state (EOS) [1] based on the Rydberg potential function [2] has been widely used [3-11] in spite of its main shortcoming regarding the extreme compression behaviour of solids in the limit of infinite pressure $[12,13]$. This shortcoming was rectified $[10,14]$ by generalizing the Vinet-Rydberg EOS. The generalized Rydberg EOS formulated by Stacey [14] can be written as

$$
P=3 K_{0} x^{-K_{\infty}^{\prime}}\left(1-x^{1 / 3}\right) \exp \left[f\left(1-x^{1 / 3}\right)\right]
$$

where $\mathrm{K}_{0}$ is the value of bulk modulus at $P=0, x=V / V_{0}$, and $K_{\infty}^{\prime}$ is the value of pressure derivative of bulk modulus at $P \rightarrow \infty$. The parameter $f$ appearing in the exponential term in equation (1) is given below

$$
f=\frac{3}{2} K_{0}^{\prime}-3 K_{\infty}^{\prime}+\frac{1}{2}=-3 K_{0} K_{0}^{\prime \prime}-\frac{3}{4} K_{0}^{\prime 2}+\frac{1}{12}
$$

where $K_{0}^{\prime}$ and $K_{0}^{\prime \prime}$ are the values of the first and the second pressure derivatives of bulk modulus, both at $P=0$. According to Stacey [12-14], $K_{\infty}^{\prime}$ is an equation of state parameter depending on the material in the same sense as $K_{0}^{\prime}$ and $K_{0}^{\prime \prime}$ are. On the basis of thermodynamic principles, it has been found $[13,14]$ that $K_{\infty}^{\prime}>5 / 3$. Stacey [14] emphasized that the generalized Rydberg EOS has the advantage of being successfully applicable to the solids under compressions as well as to the solids when they are dilated. Singh [15] has also demonstrated the usefulness of the generalized Rydberg EOS in investigating the high pressure properties of solids. In the present study we produce formulations for the higher derivative thermoelastic parameters and for the pressure derivatives of bulk modulus using the generalized Rydberg EOS and our earlier approach [16] based on the modified free volume theory of Grüneisen parameter [13,17]. Then we discuss and compare the results based on other equations of state. 


\section{Grüneisen parameter and thermoelastic properties}

The most important parameter providing connection between thermal and elastic properties is the Grüneisen parameter [18]

$$
\gamma=\frac{\alpha K_{\mathrm{T}}}{\rho C_{\mathrm{V}}}=\frac{\alpha K_{\mathrm{S}}}{\rho C_{\mathrm{P}}},
$$

where $\alpha$ is thermal expansivity, $\rho$ is density, $K_{\mathrm{T}}$ and $K_{\mathrm{S}}$ are isothermal and isentropic bulk moduli, $C_{\mathrm{V}}$ and $C_{\mathrm{P}}$ are specific heats at constant volume and constant pressure, respectively. According to the generalized free-volume formula $[13,17], \gamma$ is related to pressure $P$, bulk modulus $\mathrm{K}$ and its pressure derivative $K^{\prime}=\mathrm{d} K / \mathrm{d} P$, as follows

$$
\gamma=\frac{\left(K^{\prime} / 2\right)-(1 / 6)-(t / 3)(1-P / 3 K)}{1-2 t(P / 3 K)} .
$$

The parameter $t$ takes different values for different formulations of gamma based on different approximations reviewed by Stacey and Davis [13]. Thus $t=0$ for the Slater formula [19], $t=1$ for the formulation developed by Dugdale and MacDonald [20], $t=2$ for the free-volume formula [17], and $t=2.35$ resulted in a molecular dynamical calculation by Barton and Stacey [21]. Equation (4) can be applied to different types of solids, metals as well as insulators, because it is derived from the fundamental relationship between thermal pressure and thermal energy [18,21]. The pressure dependence or volume dependence of the Grüneisen parameter can be studied with the help of equation (4) using different equations of state [6,22,23]. The value of $t$ in equation (4) can be determined empirically by adjusting it to some known condition such as $\gamma=\gamma_{0}$ at $P=0$. The value of $t$ determined in this way has been found to be equal to 1.436 for the lower mantle and outer core [13,16]. Expressions for higher order Grüneisen parameters $q$ and $\lambda$ can be derived by taking $t$ as a constant. The final result for the third order Grüneisen parameter obtained in the present study for solids in the limit of extreme compression is independent of the parameter $t$.

By rewriting equation (4) in the following equivalent form [22]

$$
\gamma=\frac{K^{\prime}}{2}-\frac{1}{6}-\varepsilon
$$

where

$$
\varepsilon=\frac{t\left(K-K^{\prime} P\right)}{(3 K-2 t P)} .
$$

The higher order Grüneisen parameters are defined as [16,24]

$$
q=\left(\frac{\mathrm{d} \ln \gamma}{\mathrm{d} \ln V}\right)_{\mathrm{T}}=-\frac{K}{\gamma}\left(\frac{\mathrm{d} \gamma}{\mathrm{d} P}\right)_{\mathrm{T}}
$$

and

$$
\lambda=\left(\frac{\mathrm{d} \ln q}{\mathrm{~d} \ln V}\right)_{\mathrm{T}}=-\frac{K}{q}\left(\frac{\mathrm{d} q}{\mathrm{~d} P}\right)_{\mathrm{T}} .
$$

Then, the following expressions are obtained from the differentiation of equation (5)

$$
q \gamma=-\frac{K K^{\prime \prime}}{2}+K \frac{\mathrm{d} \varepsilon}{\mathrm{d} P}
$$

and

$$
\gamma q(q+\lambda)=\frac{K^{\prime} K K^{\prime \prime}}{2}+\frac{K^{2} K^{\prime \prime \prime}}{2}-K K^{\prime} \frac{\mathrm{d} \varepsilon}{\mathrm{d} P}-K^{2} \frac{\mathrm{d}^{2} \varepsilon}{\mathrm{d} P^{2}} .
$$

Equations (9) and (10) yield

$$
(q+\lambda)=-K^{\prime}-\frac{\left[\left(K^{2} K^{\prime \prime \prime} / K K^{\prime \prime}\right)-\left(2 K / K^{\prime \prime}\right)\left(\mathrm{d}^{2} \varepsilon / \mathrm{d} P^{2}\right)\right]}{\left.1-\left(2 / K^{\prime \prime}\right) \mathrm{d} \varepsilon / \mathrm{d} P\right)}
$$


where the pressure derivatives of $\varepsilon$ are obtained from equation (6) as follows

$$
\frac{\mathrm{d} \varepsilon}{\mathrm{d} P}=-\frac{\left[t K^{\prime \prime} P+\varepsilon\left(3 K^{\prime}-2 t\right)\right]}{(3 K-2 t P)}
$$

and

$$
\frac{\mathrm{d}^{2} \varepsilon}{\mathrm{d} P^{2}}=-\frac{\left[t K^{\prime \prime \prime} P+t K^{\prime \prime}+3 \varepsilon K^{\prime \prime}+2\left(3 K^{\prime}-2 t\right)(\mathrm{d} \varepsilon / \mathrm{d} P)\right]}{(3 K-2 t P)}
$$

Equations (12) and (13) can be rearranged as follows according to the terms appearing in equation (11)

$$
\frac{1}{K^{\prime \prime}}\left(\frac{\mathrm{d} \varepsilon}{\mathrm{d} P}\right)=-\frac{\left[t+\left(\varepsilon / K^{\prime \prime} P\right)\left(3 K^{\prime}-2 t\right)\right]}{[(3 K / P)-2 t)]}
$$

and

$$
\frac{K}{K^{\prime \prime}}\left(\frac{\mathrm{d}^{2} \varepsilon}{\mathrm{d} P^{2}}\right)=-\frac{\left[t\left(K^{2} K^{\prime \prime \prime} / K K^{\prime \prime}\right)+(t+3 \varepsilon) K / P+\left(3 K^{\prime}-2 t\right)(K / P)\left(2 / K^{\prime \prime}\right)(\mathrm{d} \varepsilon / \mathrm{d} P)\right]}{(3-2 t P / K)(K / P)} .
$$

Equation (6) is rearranged as follows and then used in equation (14)

$$
\frac{\varepsilon}{K^{\prime \prime} P}=\frac{t\left(K-K^{\prime} P\right)}{(3-2 t P / K) K^{2} K^{\prime \prime} P / K} .
$$

The most important results based on thermodynamics of solids $[13,25]$ are that $(1) \gamma \rightarrow \gamma_{\infty}$, a finite positive value, (2) $q \rightarrow 0$, and (3) $\lambda \rightarrow \lambda_{\infty}>0$, all at infinite pressure. For all the equations of state which satisfy $K_{\infty}^{\prime}>0$, we can write [14]

$$
K_{\infty}^{\prime}=\left(\frac{P}{K}\right)_{\infty}^{-1}
$$

\section{Pressure derivatives of bulk modulus}

Here we derive the pressure derivatives of bulk modulus up to the third order using the generalized Rydberg EOS (equation (1)), and then study the relationship between the third order Grüneisen parameter $\lambda$ and the pressure derivatives of bulk modulus. Equation (1), on differentiating with respect to $x=V / V_{0}$ and using the definition of bulk modulus, $K=-x(\mathrm{~d} P / \mathrm{d} x)$, gives

$$
\frac{K}{P}-\frac{x^{1 / 3}}{3\left(1-x^{1 / 3}\right)}=K_{\infty}^{\prime}+\frac{f}{3} x^{1 / 3}
$$

where $\mathrm{f}$ is given by equation (2). At extreme compression, $x \rightarrow 0$, equation (18) reduces to equation (17). By successive differentiations of equation (18) with respect to pressure we obtain the following expressions for $K^{\prime}=\mathrm{d} K / \mathrm{d} P, K^{\prime \prime}=\mathrm{d}^{2} K / \mathrm{d} P^{2}$ and $K^{\prime \prime \prime}=\mathrm{d}^{3} K / \mathrm{d} P^{3}$

$$
\begin{gathered}
K^{\prime}\left(\frac{K}{P}\right)-\left(\frac{K}{P}\right)^{2}=-\frac{1}{3} y-y^{2}-\frac{f}{9} x^{1 / 3} \\
\left(K K^{\prime \prime}+K^{\prime 2}\right)\left(\frac{K}{P}\right)-3 K^{\prime}\left(\frac{K}{P}\right)^{2}+2\left(\frac{K}{P}\right)^{3}=\frac{1}{9} y+y^{2}+2 y^{3}+\frac{f}{27} x^{1 / 3}
\end{gathered}
$$

and

$$
\begin{gathered}
\left(K^{2} K^{\prime \prime \prime}+4 K K^{\prime \prime} K^{\prime}+K^{\prime 3}\right)\left(\frac{K}{P}\right)-\left(4 K K^{\prime \prime}+7 K^{\prime 2}\right)\left(\frac{K}{P}\right)^{2}+12 K^{\prime}\left(\frac{K}{P}\right)^{3}-6\left(\frac{K}{P}\right)^{4} \\
=-\frac{1}{27} y-\frac{7}{9} y^{2}-4 y^{3}-6 y^{4}-\frac{f}{81} x^{1 / 3}
\end{gathered}
$$


where $y=(1 / 3) x^{1 / 3} /\left(1-x^{1 / 3}\right)$. It may be noted that $y \rightarrow 0$ as $\mathrm{x} \rightarrow 0$ at extreme compression. Then, it is also revealed from equations (20) and (21) with the help of equation (17) that $K K^{\prime \prime} \rightarrow 0$ as well as $K^{2} K^{\prime \prime \prime} \rightarrow 0$ in the limit of infinite pressure.

Equations (19) and (20) give

$$
\frac{K K^{\prime \prime}}{K^{\prime}-(K / P)}+K^{\prime}-2\left(\frac{K}{P}\right)=-\frac{\left(3 y+27 y^{2}+54 y^{3}+f x^{1 / 3}\right)}{3\left(3 y+9 y^{2}+f x^{1 / 3}\right)} .
$$

By rearranging equation (21) and then dividing by equation (20), we find

$$
\frac{N}{D}=-\frac{\left(3 y+63 y^{2}+324 y^{3}+486 y^{4}+f x^{1 / 3}\right)}{3\left(3 y+27 y^{2}+54 y^{3}+f x^{1 / 3}\right)}
$$

where the numerator $\mathrm{N}$ and denominator $\mathrm{D}$ on the left hand side of equation (23) are given as follows

$$
N=\frac{K^{2} K^{\prime \prime \prime}}{K K^{\prime \prime}}+4\left(K^{\prime}-\frac{K}{P}\right)+\frac{K^{\prime 2}}{K K^{\prime \prime}}\left(K^{\prime}-\frac{K}{P}\right)-\frac{6}{K K^{\prime \prime}}\left(\frac{K}{P}\right)\left(K^{\prime}-\frac{K}{P}\right)^{2}
$$

and

$$
D=1+\left(\frac{1}{K K^{\prime \prime}}\right)\left(K^{\prime}-\frac{K}{P}\right)\left(K^{\prime}-\frac{2 K}{P}\right) .
$$

Equations given above are presented such that they can be simplified and evaluated conveniently at infinite pressure.

\section{Extreme compression behaviour}

For solids at extreme compression $(V \rightarrow 0$, and $P \rightarrow \infty)$ we derive the following relations. Equation (22) at $P \rightarrow \infty$ gives

$$
\left[\frac{K^{2} K^{\prime \prime}}{\left(K-K^{\prime} P\right)}\right]_{\infty}=-K_{\infty}^{\prime}\left(K_{\infty}^{\prime}-\frac{1}{3}\right)
$$

Using this result (equation (26)) in equation (16) and applying the condition at $P \rightarrow \infty$, we get

$$
\left(\frac{\varepsilon}{K^{\prime \prime} P}\right)_{\infty}=\frac{-t K_{\infty}^{\prime}}{\left(3 K_{\infty}^{\prime}-2 t\right)\left(K_{\infty}^{\prime}-\frac{1}{3}\right)}
$$

Equation (14) gives the following result at infinite pressure

$$
\left[\frac{1}{K^{\prime \prime}}\left(\frac{\mathrm{d} \varepsilon}{\mathrm{d} P}\right)\right]_{\infty}=\frac{-t}{3\left(3 K_{\infty}^{\prime}-2 t\right)\left(K_{\infty}^{\prime}-\frac{1}{3}\right)} .
$$

Equations (23)-(25) with the help of equation (26) yield

$$
\left(\frac{K^{2} K^{\prime \prime \prime}}{K K^{\prime \prime}}\right)_{\infty}=-\left(K_{\infty}^{\prime}+\frac{1}{3}\right) .
$$

With the help of Equations (28) and (29), equation (15) is reduced to the following result at $P \rightarrow \infty$

$$
\left[\frac{1}{K^{\prime \prime}}\left(\frac{\mathrm{d}^{2} \varepsilon}{\mathrm{d} P^{2}}\right)\right]_{\infty}=-\frac{t\left(K_{\infty}^{\prime}+\frac{1}{3}\right)}{3\left(3 K_{\infty}^{\prime}-2 t\right)\left(K_{\infty}^{\prime}-\frac{1}{3}\right)} .
$$

The final result for the third order Grüneisen parameter $\lambda$ at $\mathrm{P} \rightarrow \infty$ is obtained from equation (11) using equations (28)-(30). It should be mentioned that the second order Grüneisen parameter q (equation (9)) tends to zero at $P \rightarrow \infty$ since $K K^{\prime \prime}, \varepsilon$ and $\mathrm{d} \varepsilon / \mathrm{d} P$ all tend to zero as it is evident from equations (20), (6) and (12), respectively. Thus, we find from equation (11) at infinite pressure

$$
\lambda_{\infty}=\frac{1}{3} .
$$




\section{Discussions and conclusions}

The third order Grüneisen parameter $\lambda$ is an important physical quantity related to pressure derivatives of bulk modulus up to the third order and, therefore, depends on the derivatives of potential energy up to the fifth order [13]. Any fundamental knowledge about $\lambda$ would provide a new powerful lead to high pressure equation of state. Thus, $\lambda_{\infty}$ is an important equation of state parameter like $K_{\infty}^{\prime}$. In addition to this, $\lambda_{\infty}$ plays a significant role in describing the volume dependence of the Grüneisen parameter and other thermoelastic properties. We have the following relationship

$$
\begin{aligned}
\lambda & =\frac{V}{q}\left(\frac{\mathrm{d} q}{\mathrm{~d} V}\right)=\left(\frac{\gamma}{\mathrm{d} \gamma / \mathrm{d} V}\right) \frac{\mathrm{d}}{\mathrm{d} V}\left[\frac{V}{\gamma}\left(\frac{\mathrm{d} \gamma}{\mathrm{d} V}\right)\right] \\
& =\left(\frac{\gamma}{\mathrm{d} \gamma / \mathrm{d} V}\right)\left[\frac{1}{\gamma}\left(\frac{\mathrm{d} \gamma}{\mathrm{d} V}\right)-\frac{V}{\gamma^{2}}\left(\frac{\mathrm{d} \gamma}{\mathrm{d} V}\right)^{2}+\frac{V}{\gamma}\left(\frac{\mathrm{d}^{2} \gamma}{\mathrm{d} V^{2}}\right)\right] \\
& =1-\frac{V}{\gamma}\left(\frac{\mathrm{d} \gamma}{\mathrm{d} V}\right)+\frac{V\left(\mathrm{~d}^{2} \gamma / \mathrm{d} V^{2}\right)}{(\mathrm{d} \gamma / \mathrm{d} V)}
\end{aligned}
$$

Burakovsky and Preston [23] have formulated the following model for the volume dependence of $\gamma$

$$
\gamma=\gamma_{\infty}+c_{1}\left(\frac{V}{V_{0}}\right)^{m}+c_{2}\left(\frac{V}{V_{0}}\right)^{n},
$$

where $c_{1}$ and $c_{2}$ are constants for a given material. The exponent $m>1$, and $n=1 / 3$. At extreme compression $V \rightarrow 0$, we have $\gamma=\gamma_{\infty}$. Equations (32) and (33) when used at infinite pressure reveal that $\lambda_{\infty}=n=1 / 3$. Thus, the result for $\lambda_{\infty}$ obtained in the present study using the generalized Rydberg EOS is consistent with the model for $\gamma(V)$ due to Burakovsky and Preston [23]. It should be mentioned that the parameters in equation (33) are likely to change for a material undergoing phase transitions. However, equation (33) can be extrapolated to extreme compression by assuming that the material remains in the same phase.

It should be pointed out that in the present formulation based on equations (5) and (6) we have taken $\varepsilon \rightarrow 0$ at $P \rightarrow \infty$ which is possible only when the denominator of equation (6) does not become zero, i.e. $K_{\infty}^{\prime}$ remains different from $2 t / 3$. When $\varepsilon \rightarrow 0$ at $P \rightarrow \infty$, equation (5) gives

$$
\gamma_{\infty}=\frac{K_{\infty}^{\prime}}{2}-\frac{1}{6}
$$

Equation (34), which represents the Slater formula at $P \rightarrow \infty$, is valid according to the analysis presented by Stacey [25]. However, in the Burakovsky-Preston model, we have $t=5 / 2$ in the extreme compression limit, and $K_{\infty}^{\prime}=5 / 3$ and, therefore, $K_{\infty}^{\prime}=2 t / 3$. Thus, according to this model $[23,26], \varepsilon$ does not become zero, and equation (34) is not a valid result. In fact, $\varepsilon$ becomes indeterminate from equation (6) at $P \rightarrow \infty$ when $K_{\infty}^{\prime}=2 t / 3=5 / 3$. It should be mentioned that Burakovsky and Preston [23] have taken the parameter $t$ to change with pressure in their formulation based on the generalized free volume theory. Thus, the values of $t$ taken by them remain between zero and $5 / 2$ respectively at $P=0$ and at $P \rightarrow \infty$.

Stacey [12-14] has mentioned that equation (34) for $\gamma_{\infty}$ results when we substitute equation (17) in equation (4). However, this is only incomplete information. Using equation (17) in equation (4) we get the following complete expression [26]

$$
\left(K_{\infty}^{\prime}-\frac{2 t}{3}\right)\left(\gamma_{\infty}-\frac{K_{\infty}^{\prime}}{2}-\frac{1}{6}\right)=0
$$

Thus, it is evident that equation (35) reduces to equation (34) under the condition when $K_{\infty}^{\prime}$ is not equal to $2 \mathrm{t} / 3$. This point is very important emphasizing the need for an analysis involving $\varepsilon$ based on equations (5) and (6). Such an analysis is also necessary for determining higher derivative parameters $q$ and $\lambda$ at finite pressures. According to Stacey and Davis [13], the Slater formula 
assumes the status of an identity supporting the validity of equation (34). There is enough evidence as reviewed by Burakovsky and Preston [23] that the maximum value of $t$ can be $5 / 2$, and, therefore, $2 t / 3$ cannot be more than $5 / 3$. Since the Stacey thermodynamic constraint reveals $K_{\infty}^{\prime}>5 / 3$, so $K_{\infty}^{\prime}$ is also greater than $2 t / 3$, and hence equation (34) is fundamentally correct.

However, we have to follow an alternative approach to find $\lambda_{\infty}$ when $K_{\infty}^{\prime}=5 / 3$. In this case the generalized Rydberg EOS (equation (1)) reduces to the following form at strong compressions when $x=V / V_{0}$ is close to zero,

$$
P=a V^{-5 / 3} \exp \left(-b V^{1 / 3}\right)
$$

where $a$ and $b$ are constants greater than zero. Equation (36) was used in the literature [27,28] as an EOS yielding accurate results at ultrahigh pressures. An analysis based on equation (36), for which $K_{\infty}^{\prime}=5 / 3$, was presented earlier [26] where the same result, $\lambda_{\infty}=1 / 3$, was obtained. It should be mentioned that the melting curves for $\mathrm{Al}, \mathrm{Ar}, \mathrm{Cu}, \mathrm{Pd}$ and $\mathrm{Pt}$ determined using $n=\lambda_{\infty}=1 / 3$ in equation (33) present close agreement with the experimental data $[23,29,30]$.

It should be emphasized that the result $\lambda_{\infty}=1 / 3$ is specific to the generalized Rydberg EOS (equation (1)) formulated by Stacey [14]. This value of $\lambda_{\infty}$ is in agreement with the BurakovskyPreston model [23] but substantially deviates from the result $\lambda_{\infty} \approx 3$ for the Earth core derived from an analysis of seismic data [13]. The seismic value of $\lambda_{\infty}$ is significantly higher than $\lambda_{\infty}=$ $K_{\infty}^{\prime 2} / K_{0}^{\prime}=1.8$ obtained by Shanker and Singh [16] from the Stacey reciprocal K-primed EOS [24] taking $K_{0}^{\prime}=5.0$ and $K_{\infty}^{\prime}=3.0$ for the Earth's core. It shows that $\lambda_{\infty}$ is very sensitive to the assumed equations and that any secure fix on it will be a very powerful constraint on equations of state in general. To achieve this objective, we need to compare the results with those based on other equations of state.

Stacey [12-14,25] has emphasized in a very convincing manner that analytical equations of state describing the behaviour of materials through all the phase transitions from zero pressure to the Thomas-Fermi state are not valid mainly because the parameters appearing in such equations cannot remain unchanged through phase transitions. The same criticism applies to equation (33) for $\gamma(V)$ due to Burakovsky and Preston [23]. However, various equations can be generalized in the same way as the Rydberg EOS, and can be extrapolated to the extreme compression by considering the material to remain in the same phase so as to satisfy equation (17). In this case the infinite pressure parameters such as $K_{\infty}^{\prime}, \gamma_{\infty}, \lambda_{\infty}$ are not the Thomas-Fermi state parameters. They are material - dependent and phase-dependent in the same sense as the zero pressure parameters $\left(K_{0}\right.$, $K_{0}^{\prime}, K_{0}^{\prime \prime}$ ) depend on the material in a given phase.

We have generalized the Holzapfel EOS (AP2) and the Hama-Suito EOS [3,22] by taking the factor $\left(V / V_{0}\right)^{-K_{\infty}^{\prime}}$ in place of $\left(V / V_{0}\right)^{-5 / 3}$, in the same way as Stacey [14] generalized the Rydberg EOS. These two equations after generalization yield the same result $\lambda_{\infty}=1 / 3$ with the help of formulation based on the modified free volume theory [16]. The generalized fourth-order PoirierTarantola logarithmic EOS [31,32] yields a different result, $\lambda_{\infty}=0$, which is not consistent with the thermodynamic considerations $\left(\lambda_{\infty}>0\right)$. The Keane EOS [33], as discussed in the Appendix, gives $\lambda_{\infty}=K_{\infty}^{\prime}$. This result is in agreement with $\lambda_{\infty} \approx 3$ [13] because $K_{\infty}^{\prime}$ is also nearly 3 for the earth's core.

The most important finding based on different equations of state discussed above is that all of them satisfy the following relationship at infinite pressure for the ratio of third- and second pressure derivatives of bulk modulus

$$
\left(\frac{K^{2} K^{\prime \prime \prime}}{K K^{\prime \prime}}\right)_{\infty}=-\left(K_{\infty}^{\prime}+\lambda_{\infty}\right) .
$$

Equation (37) is a result of detailed analysis involving $\varepsilon$ and its pressure derivatives $\mathrm{d} \varepsilon / \mathrm{d} P$ and $\mathrm{d}^{2} \varepsilon / \mathrm{d} P^{2}$. The conclusion drawn by Stacey and Davis [13] that the Slater formula becomes valid at infinite pressure gets strong support from the validity of equation (37). In fact, equation (37) can be directly obtained by differentiating twice the Slater formula for $\gamma(\varepsilon=0$ in equation (5)). Equation (11) is also directly reduced to equation (37) at infinite pressure when the pressure derivatives of $\varepsilon$ are zero. But we did not arrive at equation (37) in this manner. 
Stacey [12-14] has given two alternative statements: (i) the Slater formula becomes valid at $P \rightarrow \infty$, and (ii) all the formulae for $\gamma$ represented by equation (4) reduce to equation (34). The two statements are not equivalent. The second statement appears to be more appropriate. It is clear from equation (6) that $\varepsilon \rightarrow 0$ at $P \rightarrow \infty$ due to equation (17) for any value of $t=0,1,2$ etc. This favours the second statement. It is further substantiated by the fact that equation (11) reduces to equation (37) even when $\mathrm{d} \varepsilon / \mathrm{d} P$ and $\mathrm{d}^{2} \varepsilon / \mathrm{d} P^{2}$ are not zero at $P \rightarrow \infty$. It has been found in the present study that different equations of state satisfy the relationship

$$
\left(\frac{K^{2} K^{\prime \prime \prime}}{K K^{\prime \prime}}\right)_{\infty}=\frac{\left[\left(K / K^{\prime \prime}\right)\left(\mathrm{d}^{2} \varepsilon / \mathrm{d} P^{2}\right)\right]_{\infty}}{\left[\left(1 / K^{\prime \prime}\right)(\mathrm{d} \varepsilon / \mathrm{d} P)\right]_{\infty}}
$$

Equation (38) when used in equation (11) gives equation (37). Equation (38) is valid for all values of $t$, and, therefore, various formulae based on different values of $t$ converge to equation (37) for $\lambda_{\infty}$.

\section{Appendix}

Here we present some expressions based on the K-primed equations. Using the Stacey EOS we obtained [16]

$$
\lambda_{\infty}=\frac{K_{\infty}^{\prime 2}}{K_{0}^{\prime}}
$$

and

$$
\left(\frac{K^{2} K^{\prime \prime \prime}}{K K^{\prime \prime}}\right)_{\infty}=-\frac{K_{\infty}^{\prime}}{K_{0}^{\prime}}\left(K_{0}^{\prime}+K_{\infty}^{\prime}\right)
$$

Equations (39) and (40) exactly satisfy equation (37). Equations (25), (26) and (32) of [16] are identical with equations (13), (11) and (15) respectively, which have been written in compact forms in the present paper.

There has been a mistake in the interpretation of results [16] based on the Keane EOS [33]. The correct interpretation is as follows. For the Keane EOS we have

$$
K^{2} K^{\prime \prime}=-K_{0} K^{\prime}\left(K_{0}^{\prime}-K_{\infty}^{\prime}\right) .
$$

In the limit $P \rightarrow \infty, K^{\prime} \rightarrow K_{\infty}^{\prime}$, and, therefore, $K^{2} K^{\prime \prime}$ remains finite. Using the Keane EOS, we can also write

$$
\frac{\left(K-K^{\prime} P\right)}{K_{0}}=1-\left(K_{0}^{\prime}-K_{\infty}^{\prime}\right) \ln x+\left(K_{0}^{\prime}-K_{\infty}^{\prime}\right) \frac{P}{K} .
$$

Equation (42) gives

$$
\left(K-K^{\prime} P\right)_{\infty} \rightarrow \infty
$$

at $x=V / V_{0} \rightarrow 0(P \rightarrow \infty)$. In spite of equation (43), $\varepsilon_{\infty} \rightarrow 0$ because equation (6) can be rewritten as

$$
\varepsilon=\frac{t\left(1-K^{\prime} P / K\right)}{(3-2 t P / K)} .
$$

Equation (44) gives $\varepsilon_{\infty} \rightarrow 0$ due to equation (17). The results expressed in equations (36) and (37) of [16] are modified as follows

$$
\left(\frac{K^{2} K^{\prime \prime}}{K-K^{\prime} P}\right) \rightarrow 0
$$

Equation (45) is based on equations (41) and (43). Equation (45) substituted in equation (16) gives

$$
\left(\frac{\varepsilon}{K^{\prime \prime} P}\right) \rightarrow \infty
$$

With the help of equations (46) and (14) we get

$$
\frac{1}{K^{\prime \prime}}\left(\frac{\mathrm{d} \varepsilon}{\mathrm{d} P}\right) \rightarrow \infty \text {. }
$$


The Keane EOS gives [16]

$$
\left(\frac{K^{2} K^{\prime \prime \prime}}{K K^{\prime \prime}}\right)_{\infty}=-2 K_{\infty}^{\prime} .
$$

Equations (47) and (48) when substituted in equation (15) yield

$$
\frac{\left[\left(K / K^{\prime \prime}\right)\left(\mathrm{d}^{2} \varepsilon / \mathrm{d} P^{2}\right)\right]_{\infty}}{\left[\left(1 / K^{\prime \prime}\right)(\mathrm{d} \varepsilon / \mathrm{d} P)\right]_{\infty}}=-2 K_{\infty}^{\prime} .
$$

Finally we find from equation (11) the following result

$$
\lambda_{\infty}=K_{\infty}^{\prime}
$$

It should be mentioned that equations (48), (49) and (50) satisfy Equations (37) and (38) given in the present paper. Equations (31), (33) and (34) of [16] based on the Stacey EOS also satisfy equations (37) and (38). Not only the Keane EOS and the Stacey EOS, but also other equations, such as the Rydberg EOS, Holzapfel EOS, Hama-Suito EOS, Poirier-Tarantola EOS satisfy equations (37) and (38). It should be mentioned that equations (17), (34), (37) and (38) are quite general and they do not depend on the empirical equations of state.

Equation (38) can further be solved using the results based on equations (14)-(16) at $P \rightarrow \infty$. Thus we get from equation (38)

$$
\left(\frac{K^{2} K^{\prime \prime \prime}}{K K^{\prime \prime}}\right)_{\infty}=-2 K_{\infty}^{\prime}-\frac{1}{K_{\infty}^{\prime}}\left(\frac{K^{2} K^{\prime \prime}}{K-K^{\prime} P}\right)_{\infty} .
$$

Equations (37) and (51) give

$$
\lambda_{\infty}=K_{\infty}^{\prime}+\frac{1}{K_{\infty}^{\prime}}\left(\frac{K^{2} K^{\prime \prime}}{K-K^{\prime} P}\right)_{\infty} .
$$

We have verified that equations (51) and (52) are satisfied by the Stacey EOS, Keane's EOS and generalized Rydberg EOS. This is a remarkable finding, particularly in view of the fact that $\lambda_{\infty}$, $\left(K^{2} K^{\prime \prime \prime} / K K^{\prime \prime}\right)_{\infty}$ and $\left(K^{2} K^{\prime \prime} /\left(K-K^{\prime} P\right)\right)_{\infty}$ determined from one EOS differ much from those based on the other EOS.

\section{References}

1. Vinet P., Ferrante J., Rose J.H., Smith J.R., Geophys. Res., 1987, 92, 9319; J. Phys. Condens. Matter, 1989, 1, 1941.

2. Rydberg R., Z. Phys., 1932, 73, 376.

3. Hama J., Suito K., J. Phys. Condens Matter, 1996, 8, 67.

4. Shanker J., Kushwah S.S., Sharma M.P., Physica B, 1999, 271, 158.

5. Cohen R.E., Gulsern O., Hemley R.J., American Mineralog., 2000, 85, 338.

6. Vocadlo L., Poirier J.-P., Price G.D., American Mineralog., 2000, 85, 390.

7. Gaurav S., Sharma B.S., Sharma S.B., Upadhyaya S.C., Physica B, 2002, 322, 328; J. Phys. Chem. Solids, 2004, 65, 1635.

8. Sushil K., Arunesh K., Singh P.K., Sharma B.S., Physica B, 2004, 352, 134.

9. Singh V., Gautam A.K., Singh K.S., Physica B, 2004, 352, 164.

10. Arunesh K., Dharmendra K., Physica B, 2005, 364, 130.

11. Chauhan R.S., Singh C.P., Physica B, 2007, 387, 352.

12. Stacey F.D., Phys. Earth Planet. Inter., 2001, 128, 179.

13. Stacey F.D., Davis P.M., Phys. Earth Planet. Inter., 2004, 142, 137.

14. Stacey F.D. Rep. Prog. Phys., 2005, 68, 341.

15. Singh B.P., Physica B, 2005, 369, 111.

16. Shanker J., B.P Singh, Physica B, 2005, 370, 78.

17. Vashchenko V.Ya., Zubarev V.N., Soy. Phys. Solid State, 1963, 5, 653.

18. Anderson O.L. Equation of State of Solids for Geophysics and Ceramic Sciences. Oxford University Press, New York, 1995. 
19. Slater J.C. Introduction to Chemical Physics. McGraw, New York, 1939.

20. Dugdale J.S., MacDonald D.K.C., Phys. Rev., 1953, 89, 832.

21. Barton M.A., Stacey F.D., Phys. Earth Planet. Inter., 1985, 39, 167.

22. Holzapfel W.B., Hartwig M., Sievers W., J. Phys. Chem. Ref. Data, 2001, 30, 515.

23. Burakovsky L., Preston D.L., J. Phys. Chem. Solids, 2004, 65, 1581.

24. Stacey F.D., Isaak D.G., Geophys. J. Int., 2001, 146, 143.

25. Stacey F.D., Geophys. J. Int., 2000, 143, 621.

26. Shanker J., Singh B.P., Baghel H.K., Physica B, 2007, 387, 409.

27. Barnes J.F., Phys. Rev., 1967, 153, 153.

28. Holzapfel W.B. - In. Molecular Systems Under High Pressure, eds. Pucci R., Piccitto G. North-Holland, Amsterdam, 1991, p. 61.

29. Cheng V.M., Daniel W.B., Crawford R.K., Phys. Lett. A, 1973, 43, 109.

30. Jeong J.-W., Chang K.J., J. Phys. Condens. Matter, 1999, 11, 3799.

31. Poirier J.-P., Tarantola A., Phys. Earth Planet. Inter., 1998, 109, 1.

32. Kushwah S.S., Shrivastava H.C., Singh K.S., Physica B, 2007, 388, 20.

33. Keane A., Aust. J. Phys., 1954, 7, 322.

\title{
Властивості твердих тіл при екстремальному стисненні на основі узагальненого рівняння стану Рідберга
}

\author{
Дж.Шанкер, Б.П.Сінг, К.Джітендра \\ Фізичний факультет, Інститут природничих наук, Кхандарі, Агра Індія 282002 \\ Отримано 24 вересня 2008 р., в остаточному вигляді - 2 квітня 2009 р.
}

\begin{abstract}
Ми отримали формулювання для похідних третього порядку за тиском від об'ємних модулів і для параметрів Грюнайзена вищого порядку, використовуючи узагальнену теорію вільного об'єму та узагальнене рівняння стану Рідберга. Отримані властивості $є$ безпосередньо пов'язаними із розумінням термоелектричних властивостей твердих тіл. Показано, що параметр Грюнайзена третього порядку $(\lambda)$ в границі нескінченного тиску $\left(\lambda_{\infty}\right)$ наближається до скінченного позитивного значення, рівного $1 / 3$. Показано, що цей результат не залежить від значення похідної за тиском від об'ємного модуля при нескінченному тиску. Також обговорюються результати, отримані на основі інших рівнянь стану. Ми знайшли співвідношення, що зв'язує $\lambda_{\infty}$ та похідні за тиском від об'ємних модулів при нескінченному тиску, яке задовольняється для різних типів рівнянь стану.
\end{abstract}

Ключові слова: похідні за тиском від об'ємних модулів, параметри Грюнайзера, поведінка при нескінченному тиску, термоелектричні властивості

PACS: $65,64.10 .+h, 91.60 . F e, 46.25 .4 f, 62.20 . D, 81.40 . J j, 62.50 .-p$ 
